\title{
L'espace urbain à l'épreuve de la révolution numérique : nouvelles technologies urbaines et intelligence collective
}

\section{The digital revolution of urban space: digital urban technologies and}

\section{collective intelligence}

\author{
Anne Alombert ${ }^{1}$, Emilien Cristia $^{2}$ \\ ${ }^{1}$ Université Catholique de Lille, Lille, France, anne.alombert@univ-catholille.fr \\ ${ }^{2}$ Laboratoire MAACC, Paris, France, emilien.cristia@gmail.com
}

RÉSUMÉ. Cet article s'interroge sur la manière dont le Building Information Modeling/Management serait capable de transformer la réalisation d'un projet urbain. Quelles sont les nouvelles potentialités ouvertes par ces évolutions techniques en termes d'urbanité ? Permettent-elles d'associer les habitants à la production de leurs milieux urbains, en agençant leurs savoirs à ceux des acteurs professionnels et industriels ? Nous soutiendrons qu'une fois intégrés à des projets locaux assumant les singularités des territoires, et une fois appropriées et pratiquées par les habitants, une adaptation des pratiques de Building Information Modeling et de City Information Modeling pourraient devenir porteuses de nouvelles formes d'intelligence urbaine numérique, dépassant les écueils du modèle dominant des smart cities.

ABSTRACT. This article questions the way in which Building Information Modeling/Management technologies transform the conception and implementation of an urban project. What are the new urban possibilities opened up by these technical evolutions? Especially, can they allow new ways to associate the inhabitants to the production of their urban milieu, through the connection of their knowledge with the professional and industrial actors? We will try to show that if they are included in local projects, taking the territorial specificities into account, and if they are collectively appropriated by the inhabitants, these technologies could give way to a new form of digital urban intelligence, far beyond the dominant model of smart cities.

MOTS-CLÉS. CIM, BIM, données, ville ouverte, capacitation, contribution, intelligence urbaine.

KEYWORDS. CIM, BIM, data, open city, capacitation, contribution, urban intelligence.

Les évolutions technologiques et industrielles transforment en profondeur les modes de production, les organisations économiques et les systèmes sociaux. A travers le développement des technologies numériques, nous assistons à une révolution industrielle qui transforme à son tour la production et l'organisation du travail, tout comme les modes de construction, de transport, d'aménagement, de gestion et de vie urbains. Cette révolution s'accompagne d'une généralisation d'activités numériquement assistées qui ne se limitent plus à des secteurs industriels de haute précision (aérospatiale, aéronautique ou automobile). Ainsi, l'introduction de la démarche de Building Information Model/Modeling/Management (BIM) au secteur de la construction influence indéniablement les activités de planification, de conception, de construction et d'exploitationmaintenance du cadre bâti et paysager mais transforme aussi les savoirs, les économies et les modes de vie urbains. Le Building Information Modeling/Management désigne techniquement la modélisation d'informations concernant la construction d'un bâtiment, c'est-à-dire, l'« utilisation d'une représentation numérique partagée d'un objet bâti pour faciliter les processus de conception, de construction et d'exploitation et former une base fiable permettant les prises de décision ${ }^{1} »$. Le BIM désigne donc moins un outil ou une technologie qu'une démarche ou « une suite de processus (...) utilisés tout au long de la conception, de la construction et de l'utilisation d'un bâtiment ${ }^{2}$ », utilisant plusieurs logiciels pour faire communiquer les différents acteurs d'un projet et de capitaliser les

\footnotetext{
${ }^{1}$ https://www.iso.org/obp/ui/\#!iso:std:68078:fr

${ }^{2}$ http://www.objectif-bim.com/index.php/bim-maquette-numerique/le-bim-en-bref/la-definition-du-bim
} 
informations à chaque étape du processus de construction, de maintenance, d'exploitation et même de démolition. Cette méthode de travail s'appuie sur une maquette numérique, qui contient des données structurées et constitue ainsi «une représentation digitale des caractéristiques physiques et fonctionnelles des bâtiments ou des infrastructures ${ }^{3} »$.

La démarche de Building Information Modeling (BIM), qui concerne principalement les infrastructures et les bâtiments, promet de se décliner à l'échelle urbaine et de se généraliser sous la forme de projet de City Information Model-Modeling-Management (CIM), qui nous intéresse plus particulièrement. Même si le concept de « $\mathrm{CIM}$ » est aujourd'hui principalement utilisé à des fins promotionnelles à défaut de relever d'une réelle pratique, la modélisation numérique d'informations urbaines apparaît néanmoins comme une tendance pouvant raisonnablement s'imposer comme une activité liée à la fabrique et la gestion de la ville. Telle que nous l'entendons, la démarche de City Information Modeling/Management correspond à une extension des pratiques et technologies BIM à un ensemble de bâtiments, un quartier, une ville ou un territoire, grâce à la mobilisation d'une/des maquette(s) numérique(s) potentiellement capable de conserver et de traiter ensemble des informations numériques attachées à différents composants de l'espace urbain, et de faire communiquer ainsi différents acteurs dans la conception, la construction et l'exploitation de cet espace. Alors que lesdites smart cities sont en pleine croissance, la pertinence d'une maquette numérique CIM pourrait se justifier par sa capacité à articuler les informations concernant les bâtiments avec les technologies de l'information et de la communication caractéristiques des « villes intelligente » (capteurs électroniques, Internet des Objets, Cloud Computing, etc.) qui permettent la collecte des données et la gestion des flux urbains en temps réel (gestion des réseaux comme l'eau, l'énergie, l'éclairage, les télécommunications, le wifi, gestion du trafic, du stationnement, des déchets, de la pollution, etc.).

Si l'« informatisation des villes ${ }^{4} »$ s'opère généralement au nom de l'optimisation de la gestion urbaine, de l'augmentation de la fluidité des déplacements (contrôle des stationnement et du trafic) ou de l'amélioration de la qualité de vie des citoyens (mesure de la pollution, économie d'énergie, gestion des déchets), les recherches récentes en sciences humaines et sociales (géographie, sociologie, économie, philosophie) n'ont cessé de remettre en cause le modèle dominant des smart cities. Les critiques portent tout d'abord sur la propriété des données Aux yeux des Big Brother Awards $\underline{5}$, les smart cities constituent une grave violation de la vie privée des citoyens : «presque toutes ces données sont recueillies et utilisées sans que personne ne leur ait donné la permission. De plus, il est pratiquement impossible d'autoriser ceci, étant donné qu'il n'est pas clair de quelles données il s'agit exactement. Il est également difficile de savoir à quelles fins les données sont recueillies et utilisées. ${ }^{\circ} »$. De nombreux chercheurs voient aussi dans le projet des smart cities l'imposition de solutions technologiques standardisées ignorant les spécificités locales des territoires et demeurant inappropriables par les habitants ${ }^{7}$. A défaut d'être «urbanisées» (adaptées aux caractéristiques du territoire, pratiquées par les habitants et encadrées par des dynamiques culturelles et sociales), les technologies numériques pourraient bien devenir «désurbanisantes»: destructrices de l'urbanité au sens de la singularité territoriale et architecturale, mais aussi et surtout, de la civilité, de l'affabilité, de

\footnotetext{
${ }^{3}$ http://www.objectif-bim.com/index.php/bim-maquette-numerique/le-bim-en-bref/la-definition-du-bim

${ }^{4}$ G. Dupuy, L'informatisation des villes, Paris, PUF, 1992.

${ }^{5}$ «Les Big Brother Awards ou BBA (en français, Prix Big Brother), est une cérémonie de remise de prixà destination '[des] gouvernements et [des] entreprises... qui font le plus pour menacer la vie privée' . La cérémonie est organisée par l'association Privacy International : voir page Wikipédia consacrée au Big Borther Awards (https://fr.wikipedia.org/wiki/Big_Brother_Awards).

${ }^{6}$ Voir page Wikipédia consacrée à la ville intelligente (https://fr.wikipedia.org/wiki/Ville_intelligente).

${ }^{7}$ Parmi les recherches récentes, on peut citer l'analyse du « smartness mandate " par O. Halpern, l'analyse des "villes censurées » par S. Sassen, l'analyse du " solutionnisme technologique » par E. Morozov, ou encore les réflexions de B. Stiegler dans La société automatique t. 1 L'avenir du travail concernant la « prolétarisation » des habitants par les dispositifs technologiques.
} 
la solidarité, qui rendent une ville habitable et vivable - grâce auxquelles les habitants vivent en bonne « intelligence » dans leur milieu urbain.

En dépit des discours promotionnels légitimant les smart cities, ces hypothèses critiques se voient progressivement confirmées par des faits réels : depuis les nuisances engendrées par les applications GPS $^{8}$ jusqu'à la contestation citoyenne du projet de smart city futuriste mené par Google à Toronto ${ }^{9}$ ou à l'utilisation de l'Internet des Objets et des technologies de reconnaissance faciale pour surveiller et contrôler les mouvements sociaux ${ }^{10}$. Partout, les préoccupations des habitants (usages pratiques de la ville, souveraineté politique, intérêts économiques, relations sociales, libertés civiques) semblent entrer en conflit avec le fonctionnement souvent inaccessible et centralisé des systèmes technologiques, dont ils se sentent de plus en plus exclus et captifs. La question se pose donc de savoir comment remettre les habitants au cœur des processus de conception, de construction et de décision qui sont aux principes des évolutions de leurs habitats et milieux urbains. La démarche CIM, articulée aux technologies BIM, peut-elle ouvrir de nouvelles possibilités dans cette direction?

L'élaboration d'un projet CIM implique la participation des différents acteurs du projet urbain (maître d'ouvrage, ingénieur, architecte, paysagiste, promoteur immobilier mais aussi habitants et usagers), nécessite la mise en commun et l'agencement d'informations hétérogènes, autorise la visualisation du projet urbain sous forme de maquette numérique mais semble aussi pouvoir offrir des potentialités de publication et de transmission des savoirs urbains capables d'être collectivement débattus et discutés. La démarche CIM pourrait, sous réserve que l'on prenne le temps d'en construire les conditions d'existence, constituer un support permettant d'assister des prises de décision collectives et d'associer les habitants à la transformation numérique de leurs territoires, concrétisant ainsi un «droit à la ville ${ }^{11}$ numérique » de façon à veiller à ce que l'espace urbain et les données qui lui sont rattachées conservent une utilité publique et de bien commun.

Quels sont les enjeux économiques, urbanistiques et anthropologiques de ces mutations ? Comment articuler les pratiques BIM et CIM et à quels types de transformations peut-on s'attendre en termes de conception, de production et de construction des espaces urbains? Quelles pourraient être les conséquences de cette révolution urbaine sur les modes d'habitation et d'implication des citoyens dans le devenir de leur ville? A quelles conditions pourraient-elles générer de nouvelles formes d'urbanité contributive et d'intelligence collective, fondée sur les savoirs, toujours singuliers et locaux des habitants? Telles sont les questions auxquelles nous tenterons de répondre, afin de comprendre comment les technologies BIM et la démarche CIM pourraient permettre de relever les défis ouverts par l'informatisation des villes, en générant un nouveau type d'intelligence urbaine contributive.

Un bref retour historique nous permettra tout d'abord d'interroger la manière dont les technologies numériques transforment les différentes fonctions urbaines (conception, construction, gestion, habitation, déplacement, orientation) à travers la démarche CIM (I.). Nous tenterons ensuite de mettre au jour les risques engendrés par l'automatisation de la construction et de la gestion urbaine (II.), mais aussi, et surtout, les potentialités contributives de ces nouvelles technologies une fois adoptées par les habitants, en coopération avec les acteurs économiques, politiques et professionnels du territoire (III.).

\footnotetext{
${ }^{8}$ On pourra par exemple consulter la recherche de P. Courmont sur les effets que peut avoir les applications de type Waze sur les politiques de régulation du trafic autoroutier, dont on entraperçoit certains effets sur les habitants notamment dans le cas de

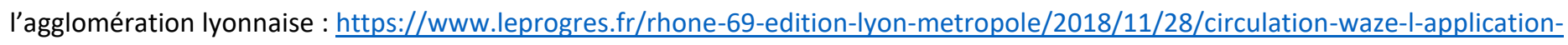
qui-agace-les-riverains

${ }^{9}$ A ce sujet, voir l'article de J. Sadowski sur la gestion d'un quartier de Toronto par Alphabet, la filiale de Google consacrée aux smart city : https://www.theguardian.com/commentisfree/2017/oct/24/google-alphabet-sidewalk-labs-toronto

${ }^{10}$ A ce sujet on pourra consulter le travail de P. Velhge qui traite de la surveillance et la gestion sociale chinoise par l'intermédiaire de l'Internet des Objets.

${ }^{11}$ H. Lefebvre, Le droit à la ville, Paris, Anthropos, 1968.
} 


\section{Etat des lieux : BIM, CIM, penser la nouvelle révolution urbaine}

Les technologies BIM provoquent des mutations fondamentales du point de vue des activités de conception, de construction et d'exploitation d'un bâtiment mais c'est à l'ensemble des activités d'aménagement et de gestion des urbanités que la démarche CIM devrait s'appliquer. Le passage de l'échelle du bâtiment à celle du système urbain dans son ensemble implique de nouvelles problématiques qu'il conviendra de dégager, tout en les connectant avec les enjeux des technologies BIM actuelles et les spécificités des futures technologies CIM. Pour envisager la complexité des mutations qu'impliquent les technologies BIM et CIM du point de vue de l'industrie du bâtiment, des métiers, des pratiques, mais aussi des habitats et des modes de vie, il apparaît essentiel de les réinscrire au sein de l'histoire des évolutions industrielles et urbaines, et en particulier, au sein du processus de globalisation des échanges qui s'amorce dès le Moyen-Age et se poursuit encore aujourd'hui ${ }^{12}$.

\subsection{Retour historique sur les transformations industrielles de l'espace urbain}

La compréhension de la révolution urbaine contemporaine ne peut être appréhendée qu'à condition d'être réinscrite dans l'histoire des transformations technologiques, industrielles et économiques, qui engendrent des reconfigurations du bâti, des infrastructures et de l'urbanité. Depuis les premiers villages agricoles jusqu'aux «smart cities », les transformations industrielles et économiques sont déterminantes quant à l'organisation tant spatiale que temporelle des modes de vies : elles s'expriment à travers les évolutions des paysages et des rythmes urbains.

Les premiers villages apparaissent avec l' «industrie» néolithique (outillage en pierre) et le développement des techniques d'agriculture et d'élevage, qui conduisent à la sédentarisation des groupes humains. L'invention de l'écriture bouleversera ensuite l'organisation spatiale et institutionnelle de la cité grecque, centrée autour de l'Agora et du Bouleutérion, qui constituent les premières formes d'espaces publics ${ }^{13}$. Au Moyen-Age, la révolution énergétique (déploiement de moulins et développement de la mécanique) transforme les techniques de production et de construction, et réorganise radicalement la cité : le bourg se structure autour du développement du compagnonnage et des guildes, qui ordonnent les métiers et les secteurs de production, alors que les serfs se constituent peu à peu en un nouveau type de main d'œuvre.

$\mathrm{Au}$ XIXème siècle, l'industrialisation de la production (machinisme, intensification de la division industrielle du travail et paternalisme industriel), le développement de la sidérurgie et des réseaux de transports et de télécommunication (voies ferrées, télégraphe) reconfigurent profondément les morphogenèses urbaines (grands magasins, diffusion de la presse et de la « réclame », développement de l'architecture et de l'urbanisme de type haussmannien) ainsi que les relations entre les villes qui constituent alors les principaux lieux de production et de consommation.

Le XXème siècle, caractérisé par l'industrie du pétrole et de l'automobile comme par les industries culturelles, fait à son tour émerger des spécificités typiques en matière de développement et d'aménagement urbain. La charte $d$ 'Athènes ${ }^{14}$ traduit dans l'organisation de la ville l'influence de d'une pensée taylorisée segmentant les quartiers selon les activités (la vie, le travail, les loisirs et les transports). Les formes de réticulations évoluent à mesure que se développent les réseaux routier et autoroutier ainsi que les réseaux hertziens de télédiffusion: deux types d'infrastructures qui contribuent au développement des grandes surfaces, centre commerciaux et hypermarchés, rendus accessibles aussi bien par les voitures individuelles que par la publicité télévisée. Inscrites dans le

\footnotetext{
${ }^{12}$ F. Braudel, La dynamique du capitalisme, Paris, Flammarion, 1977.

13 J.-P. Vernant, "Espace et organisation politique en Grèce ancienne », in Annales. Economies, sociétés, civilisations. 20 année, N. 3 , 1965.

${ }^{14}$ Le Corbusier, Charte d'Athènes, 1933. La ville de Chandigarh, pensée pour l'automobile et découpée en secteurs d'activités constitue une bonne illustration résultant d'une planification compartimentée des activités humaines.
} 
processus de globalisation des échanges, ces évolutions conduiront à la constitution de villes globales ${ }^{15}$ telles que New York, Londres ou Tokyo qui occupent aujourd'hui des fonctions stratégiques de premier plan dans la mondialisation, et organisent les flux à l'échelle internationale. Ces «villes néolibérales » repoussent sans cesse leurs habitants (et plus précisément leurs logements) en périphérie, afin d'organiser leur cœur autour de sièges sociaux, de centres commerciaux et de parcs.

\subsection{La révolution urbaine numérique : BIM, CIM et informatisation des villes}

Avec le développement des technologies numériques et du capitalisme de plateforme ${ }^{16}$, ces tendances à la néolibéralisation des villes se sont accentuées, aboutissant à des formes urbaines nouvelles parfois assimilables à de véritables «villes sièges sociaux ». Bien que leurs infrastructures soient totalement distribuées à travers la planète, les géants du Web (Google, Apple, Facebook, Amazon, Microsoft) sont des entreprises particulièrement centralisées, notamment dans les sièges sociaux de la Silicon Valley qui constituent des centres névralgiques (recherche, bureaux d'étude, stratégie et gouvernance) où tout est décidé en temps réel quel que soit le point de la planète concerné ${ }^{17}$. Outre ces effets de concentration économique et de management pyramidal, la révolution industrielle numérique a aussi de nombreuses conséquences sur les modes de construction, de transport, d'aménagement, de gestion, de déplacement et de vie urbains.

Sur les modes de vie d'abord, dans la mesure où les smartphones et objets connectés ont envahis les espaces publics et privés : l'interconnexion permanente et planétaire des individus permet la traçabilité de leurs activités et leur traitement par le calcul intensif des algorithmes. C'est cette captation et ce traitement statistique des données en temps réel qui rend par exemple possible le fonctionnement des systèmes de géolocalisation (telles les applications GPS comme Waze, Wego, MapFactor, etc.), qui modifient en profondeur les systèmes d'orientation et les manières de se déplacer. Cette nouvelle techno-économie des données se décline plus spécifiquement à l'échelle urbaine, à travers l'informatisation des villes, que le marketing désigne sous le nom de «smart cities » : les technologies numériques de l'information et de la communication (capteurs, Cloud computing, internet des objets) sont ainsi mises au service de la gestion en temps réel des flux urbains (eau, transport, éclairage, stationnement, etc.) en vue d'améliorer la performance des services et de favoriser la sobriété énergétique.

Néanmoins, la révolution urbaine contemporaine ne se limite pas à ces nouveaux modèles de smart cities, mais se caractérise aussi par des mutations industrielles plus profondes dont les enjeux demeurent encore trop peu analysés. La programmation, la conception architecturale, la modélisation urbaine et la construction des habitats entrent en pleine mutation à travers les technologies de Building Information Modeling. En effet, l'utilisation de logiciels pour produire une représentation numérique des bâtiments partagée par plusieurs acteurs, ainsi que le suivi de BIM implique de développer de nouvelles méthodes de travail et de nouvelles compétences: des corps de métiers auparavant non communiquant se voient dans l'obligation de coopérer, l'usage de nouveaux logiciels implique de nouveaux apprentissages, la répartition des tâches au sein des acteurs de la construction est modifiée et les responsabilités sont redistribuées, au point qu'une nouvelle fonction, celle de «BIM manager » a même vu le jour ${ }^{18}$. La démarche CIM, qui promet la création de maquettes numériques urbaines et la constitution de bases de données à l'échelle d'un quartier ou d'une ville, devrait faire émerger de nouveaux supports d'information et de visualisation pour la gestion de l'espace urbain. La fabrication

\footnotetext{
${ }^{15}$ S. Sassen, The Global City: New York, London, Tokyo. Princeton, Princeton University Press, 1991.

${ }^{16}$ N. Srnicek, Capitalisme de plateforme. L'hégémonie de l'économie numérique. Paris, Lux, 2018.

${ }^{17}$ O. Landau, « La production, moteur de la révolution urbaine », Paris,

${ }^{18}$ E. Cristia, P.-P. Zalio et F. Guéna (2019), « Fabriquer une maquette numérique BIM et reconsidérer le projet architectural », in Le $B I M$ entre recherche et industrialisation, Paris, Eyrolles.
} 
de ces nouveaux supports impliquera nécessairement la coopération des acteurs du projet urbain (maître d'ouvrage, ingénieur, architecte, paysagiste, promoteur immobilier mais aussi habitants et usagers) ainsi que la transformation de leurs manières de travailler.

Les évolutions des savoirs, des métiers et des pratiques vont de pair avec des évolutions économiques et industrielles toute aussi profondes. Si en France l'industrie du bâtiment reste encore largement détenue par plusieurs majors du BTP (Vinci, Bouygues, Eiffage), aux États-Unis, on assiste aujourd'hui à l'émergence de start-up dans le secteur du bâtiment, capables de menacer la position des acteurs classiques en tirant profit du numérique pour informatiser toutes les étapes de la construction ${ }^{19}$. La start up de «construction hors-site » Kattera considère par exemple pouvoir livrer des bâtiments préfabriqués ou de salles de bains en kit indépendamment de toute activité économique et professionnelle sur le territoire. Soit autant d'habitats standardisés produits dans des usines délocalisées conduisant certains journalistes à identifier un nouveau phénomène d' « ubérisation » ou une « disruption» de l'industrie du bâtiment.

\section{Les risques : automatisation, standardisation et prolétarisation}

La disruption correspond plus généralement à une transformation du système technique, qui engendre un désajustement par rapports aux systèmes sociaux existants. Dans notre cas, la numérisation des technologies de construction ou de gestion urbaine et l'informatisation des infrastructures bouleverse les organisations sociales et les normes collectives constitutives de l'urbanité traditionnelle: alors que les objets connectés et télécommunicants transforme les relations des habitants entre eux et avec leurs environnements (géolocalisation, accès à l'électricité, à l'eau, à l'énergie), le fonctionnement des technologies et outils BIM et CIM conditionne et configure quant à lui les relations entre les acteurs et les modes de gestion des projets. De plus, l'ensemble de ces transformations se produisent dans un contexte de crise climatique majeure. Dès lors, elles demandent donc à être aussi considérées du point de vue de leurs enjeux environnementaux, urbanistiques, technologiques, industriels, anthropologiques et sociétaux.

\subsection{La question des données BIM et CIM dans le contexte du capitalisme numérique}

Pour considérer les risques que peuvent soulever la numérisation des pratiques de construction et l'utilisation de ces nouvelles technologies, il nous faut d'abord essayer de circonscrire les principaux enjeux liés à la réalisation d'un projet BIM. Pourquoi cet acronyme est-il soudainement devenu indispensable à la construction? Ce sont certainement les arguments économiques qui nous permettent ici de comprendre l'efficience de ces technologies ${ }^{20}$. En effet, lorsque l'on considère l'approche en coût global d'un bâtiment (coût de conception + coût de construction + coût d'exploitation et maintenance), la démarche BIM promet prospectivement, grâce à la base de données que représente la maquette numérique, de faire de substantielles économies sur le coût d'exploitation-maintenance dont on considère qu'il représente environ $75 \%$ du coût global. Dès lors, au-delà des éventuels avantages pratiques que peut avoir la modélisation de cette maquette numérique pour la conception et le chantier, c'est le potentiel d'utilisation et la valorisation que peuvent avoir ces données qui intéresse, in fine, les

\footnotetext{
${ }^{19}$ « Aux Etats-Unis, la start-up Katerra, fondée en 2015, et qui entend révolutionner le business model de la construction en intégrant et en informatisant toutes les étapes, reste adulée pour sa progression fulgurante - elle décroche des contrats et rachète des cabinets d'architectes ou des entreprises générales dans tout le pays-, et choyée par les plus grands bailleurs de fonds internationaux. Et à ce jour, Katerra apparaît toujours comme l'étoile montante, le fleuron d'une révolution high tech dans le vieux secteur de la construction américaine. » https://www.lemoniteur.fr/article/aux-etats-unis-premiers-contretemps-pour-la-start-upkaterra.1988979

${ }^{20}$ Nous n'ignorons pas pour autant que comme toutes les innovations technologiques, les technologies BIM ont fait l'objet d'un ensemble d'effets d'annonce et de lobbying auprès des pouvoirs publics et se sont aussi développées conformément à des logiques de marketing et de fixation des prix. V. Mangematin (1993). Compétition technologique : les coulisses de la mise sur le marché. Annales des Mines.
} 
gestionnaires et les exploitants. En ce sens, il nous semble que la spécificité d'une démarche BIM n'est donc pas tant d'utiliser un type logiciel, ou de suivre certains processus, mais relève plutôt de la construction de données rattachées à des éléments bâtimentaires, urbains ou paysagers, ayant une capacité descriptive de l'état ou de l'usage dans lequel il est.

Concernant la construction de la ville et la démarche CIM, on comprend alors qu'à chaque nouveau projet BIM qui sort de terre son «avatar» ou «jumeau numérique» et leurs données peuvent représenter une forte valeur économique pour les marchés de la donnée et des services numériques. Les données d'une ville peuvent déjà être valorisées selon leur capacité à représenter une situation ou l'état d'une certaine réalité. Par exemple, les balises GPS des smartphones traités par des applications nous renseignent sur le trafic autoroutier, les données contenues dans les puces RFID des arbres parisiens permettant leur géolocalisation et l'historique des interventions dont ils ont été l'objet ${ }^{21}$, ou encore les informations relatives à la consommation d'eau ou d'électricité d'un quartier. Les start-ups ont aussi pris conscience de la quantité de données produites par les villes elles-mêmes, et de la ressource que cela représente pour pénétrer un marché de la production et exploitation de données bâtimentaires et urbaines.

\subsection{Les risques de la gouvernementalité algorithmique et du solutionnisme technologique}

Dès lors, il n'est plus possible d'omettre que la production des données à venir comme leur gestion s'inscrira dans un capitalisme numérique notamment contrôlé par les entreprises et plateformes numériques $^{22}$, dont on mesure encore mal les logiques de fonctionnement et l'étendue de l'emprise, mais qui soulève déjà un certain nombre d'enjeux du point de vue des droits civiques et de la citoyenneté. Un récent rapport publié par Amnesty Internationa ${ }^{23}$ souligne que le modèle économique des géants du web constitue une menace non seulement pour la vie privée des utilisateurs, mais aussi pour leur liberté d'expression, d'opinion et de pensée. C'est donc la vie politique qui est menacée : rappelons qu'avant de devenir une confrontation médiatique entre partis, la politique désigne fondamentalement la vie de la polis ou de la cité, c'est-à-dire la vie collective d'une communauté humaine, regroupant des sujets libres et autonomes partageant un certain nombre de règles communes qui leur permettent de se comporter de manière civilisée dans une localité.

Or, c'est précisément cette normativité politique qui se voit menacée par ce que A. Rouvroy et $\mathrm{T}$. Berns décrivent comme une «gouvernementalité algorithmique ${ }^{24}$, qui s'exerce à travers les algorithmes en téléguidant les comportements des individus sur la base de la récolte de leurs traces numériques, indépendamment de leurs consciences et de leur volonté. Selon J. Sadowski, les fondements de la démocratie se trouvent ainsi menacés par l'imposition des technologies «smart» aux localités urbaines: la ville connectée risque ainsi de devenir une plateforme automatisée, gérée à distance par les entreprises de la Silicon Valley, qui n'ont jamais été mandatées ou élues par les habitants mais qui se substituent néanmoins aux représentants et aux institutions politiques en prenant le contrôle de leurs quartiers pour expérimenter des «écosystèmes innovants » censés optimiser les flux et rétablir les objectifs de croissance économique ${ }^{25}$.

\footnotetext{
${ }^{21}$ A. Picon (2016). « L'avènement de la ville intelligente ». Sociétes, $n^{\circ} 132(2)$, 9-24.

${ }^{22}$ Notamment Google, Apple, Facebook, Amazon et Microsoft, aussi désignées par l'acronyme GAFAM.

${ }^{23}$ Amnesty International, "Surveillance giants: How the business model of Google and Facebook threatens human rights ", https://www.amnesty.org/en/documents/pol30/1404/2019/en/.
}

\footnotetext{
${ }^{24}$ A. Rouvroy et T. Berns, « Gouvernementalité algorithmique et perspectives d'émancipation. Le disparate comme condition d'individuation par la relation ? ", Réseaux, 2013/1 ( $\left.n^{\circ} 177\right)$.
}

25 J. Sadowsky, https://www.theguardian.com/commentisfree/2017/oct/24/google-alphabet-sidewalk-labs-toronto 
Ces pratiques correspondent à l'idéologie des plateformes numériques, que E. Morozov décrit comme le «solutionnisme technologique ${ }^{26}$ et que O. Halpern décrit comme le «smartness mandate $»^{27}$ et qui désigne la tendance à vouloir imposer des systèmes standards et automatisés pour résoudre les tensions sociales et les problèmes écologiques, alors même que ceux-ci sont toujours spécifiques aux territoires et aux habitants concernés. Les « solutions » technologiques, intelligentes ou innovantes viennent alors se substituer à la délibération citoyenne et aux pratiques de coopération, créant parfois de nouvelles difficultés dans la vie quotidienne des habitants. L'application Waze (application GPS permettant de calculer les itinéraires les plus rapides) a ainsi généré la colère des habitants de l'agglomération lyonnaise, car les "conseils » délivrés par l'application engendrait des embouteillages dans le centre-ville, menaçait la sécurité des enfants et perturbaient les rythmes de vies $^{28}$ : dans ce cas précis, on constate que les calculs statistiques effectués par les algorithmes demeurent incapables de prendre en compte les enjeux des contextes locaux et deviennent donc parfois nuisibles pour les habitants.

\subsection{Industries du bâtiment et industries du logiciel dans le contexte de la disruption}

Ces risques politiques, qui se manifestent au niveau de la vie collective des habitants, ne sont pas indépendants de transformations industrielles, qui se manifestent au niveau des entreprises et de la vie économique des territoires. En effet, dans un contexte où la donnée prend de plus en plus d'importance et de valeur, où les pratiques BIM se multiplient (et opèrent justement la création massive de nouvelles données), il n'est pas étonnant de constater que l'industrie du logiciel et du bâtiment, qui se côtoient pourtant depuis plusieurs décennies, semblent faire face à une concurrence d'un nouvel ordre. En effet, les données produites par l'industrie du bâtiment incarnent et décrivent à la fois l'expertise et les savoir-faire de leurs auteurs. Or puisqu'elles sont produites grâce à des logiciels privés et/ou stockées sur des plateformes en ligne, ces données et les savoirs qu'elles contiennent deviennent partagées voire détenues par l'industrie du logiciel. L'industrie du bâtiment entre ainsi en concurrence avec l'industrie du logiciel lors de la conception-construction de ces données mais aussi avec l'industrie des télécommunications, concernant leur exploitation et leur gestion.

L'exemple de la start-up Katerra qui a reçu en 2016 l'apport de 865 millions de dollars de la part du groupe SoftBank (réputé pour son investissement dans le secteur des hautes technologies numériques et disruptives) est à ce sujet édifiant. En effet, afin d'industrialiser la filière du bâtiment, Katerra adopte une démarche «très similaire à Uber, Airbnb ou WeWork » et «se dit capable de vendre un bien immobilier en 30 jours - de la conception à la remise des clés ${ }^{29}$. Pour réaliser une telle prouesse, l'entreprise semble s'appuyer sur les technologies BIM, F2F et de robotisation afin d'automatiser l'ensemble de sa production et de pouvoir ainsi produire des «immeubles en kit » à la manière des célèbres mobiliers IKEA.

Ce type d'exemple pose plusieurs questions. Il suggère tout d'abord une emprise croissante des industriels sur les concepteurs, capables d'imposer leurs produits via des bibliothèques d'objets. Se profile ainsi une tendance à la standardisation des bâtis et équipements urbains (livraison des mêmes prototypes préfabriqués indépendamment des besoins et des conditions territoriales spécifiques), qui provoquerait nécessairement une standardisation des modes de vies, au détriment des savoirs vivre et des savoir-faire locaux des habitants. Se pose aussi la question de l'« uberisation » possible de la filière

\footnotetext{
${ }^{26}$ E. Morozov and F. Bria (2015). Ripensare la smart city, Torino, Codice Ed. ; E. Morozov (2013). Pour tout résoudre, cliquez ici. Les aberrations du solutionnisme technologique. Paris, FYP Editions.

${ }^{27}$ O. Halpern, R. Mitchell et B. Dionysius Geoghegan (2017). "The smartness mandate. Notes towards a critique », in Grey Room, N. 68, p.106-12.

${ }^{28} \mathrm{https}$ ://www.leprogres.fr/rhone-69-edition-lyon-metropole/2018/11/28/circulation-waze-l-application-qui-agace-les-riverains
} 
du bâtiment avec les dangers que cela pourrait engendrer pour les économies locales : la start up Katerra prétend maîtriser le chantier à $100 \%$ et de manière indépendante d'autres sociétés ou partenaires, et tend ainsi à court-circuiter les entreprises et activités économiques locales, mais aussi à ignorer les enjeux écosystémique propres aux territoires (les matériaux disponibles, les conditions climatiques, les dimensions écologiques). Ces effets nocifs des entreprises délocalisées reposant sur des technologies de plateformes pour les territoires ont déjà été remarquées dans le cas d'Amazon ${ }^{30}$ : c'est ce que F. Pasquale décrit comme une substitution de la "souveraineté territoriale » par la «souveraineté fonctionnelle» des plateformes, qui menacent les économies locales.

\section{Les promesses : villes ouvertes et contribution des habitants}

Face aux risques politiques que représente le management pyramidal de la ville par les entreprises de la Silicon Valley ou aux risques économiques que représentent la production délocalisée et la livraison de bâtiments préfabriqués et standardisés, il nous semble néanmoins que les technologies BIM et CIM présentent de nouvelles potentialités, susceptibles de favoriser une gestion contributive de la ville et des pratiques de co-conception associant les habitants à la production de leurs milieux urbains, en partenariat avec des acteurs économiques et industriels locaux. Les dispositifs techniques ne sont jamais de simples outils univoques : leurs normes de fonctionnement internes configurent les comportements individuels et les rapports sociaux des usagers ${ }^{31}$. Pour qu'ils puissent devenir porteurs de nouvelles potentialités pour les groupes humains, et non constituer des menaces pour les individus et leurs environnements, il est nécessaire que ces dispositifs techniques soient socialisés, c'est-à-dire, pratiqués dans le cadre de projets collectifs porteurs de sens par des citoyens qui comprennent leur fonctionnement, et non pas seulement utilisés selon des modes d'emplois prescrits par le marketing, par des usagers qui ignorent tout de leurs enjeux.

\subsection{La maquette numérique, entre professionnels et publics : vers un nouveau génie urbain ?}

Soulignons tout d'abord que l'utilisation généralisée de maquettes numérique offre de nouvelles opportunités communicationnelles avec les différents acteurs du territoire, notamment en permettant de procéder à de la modélisation orientée objet et de suivre certains processus collaboratifs sur le long terme. Les données géométriques et descriptives des projets urbains deviennent de plus en plus accessibles et compréhensibles, autorisant ainsi plus aisément la possibilité pour les acteurs locaux d'accompagner les processus d'évolution des bâtiments. De plus, la construction collective d'une maquette numérique entraîne d'un point de vue technique une unification des logiciels métiers sous une même interface. Ceci a pour conséquence de déplacer les frontières professionnelles et notamment de mettre en relation les métiers de la construction et de l'urbanisme, afin de réinscrire les projets de construction dans les contextes locaux et de les penser en lien avec la production des espaces publics et avec les conditions écologiques. Les technologies de BIM et CIM, en tant qu'elles permettent un nouvel agencement entre les différents corps de métiers, ouvrent donc de nouvelles possibilités de coopération interprofessionnelles.

Plus fondamentalement, ces démarches pourraient permettre d'associer des habitants non-experts à la production de leurs milieux urbains : à condition qu'ils comprennent et maîtrisent les enjeux de ces technologies. Les habitants pourraient être conduit à produire ou examiner des données quantitatives ou qualitatives leur permettant de défendre un avis capable d'être entendu par des experts. On pourrait par exemple imaginer des systèmes de visualisation nouveaux permettant d'accueillir les commentaires

\footnotetext{
${ }^{30}$ Amazon fait régulièrement l'objet de multiples tractations à l'encontre de la précarisation de ses salariés, de l'impact de leurs entrepôts sur le développement des zones rurales ou encore les leviers d'impunité fiscale dont elle dispose.

${ }^{31}$ M. Akrich, "Comment décrire les objets techniques ? ", in Techniques et culture, 49-64, 1987. M. Akirich soutient que les outils techniques se définissent comme le rapport entre les dispositifs qu'ils incarnent et l'ensemble des usages que " remplissent » ces dispositifs.
} 
ou les expériences des habitants, selon un certain nombre de catégories et de règles explicitement délibérées. On pourrait imaginer, au même titre que la maquette architecte ou que la maquette ingénieur, une maquette habitant : il s'agirait alors de se demander de quels types de données elle pourrait être constituée. Que savent les habitants de leurs milieux urbains? Quelles données ont-ils à partager? Que disent les données qu'ils produisent de leurs manières concrètes de vivre ? B. Chaudet, M. Patrascu et J-L Bouillon observent par exemple l'importance de «feedbacks» concernant l'utilisation des logements sociaux et la nécessité de mettre en places des «données locataires » qui puissent permettre à la maquette numérique de devenir un dispositif de médiation auprès des locataires $^{32}$.

On pourrait même aller un peu plus loin en se demandant comment les habitants pourraient participer à l'élaboration de maquettes numériques. Pourraient-ils ainsi entrer en discussion avec des professionnels de la construction, de l'urbanisme et de l'architecture non plus seulement en utilisant, mais en pratiquant ces nouveaux logiciels de modélisation ? Comme le suggère B. Stiegler ${ }^{33}$, il semble que la robotisation et l'automatisation des activités de construction pourraient aussi libérer du temps pour l'invention de nouveaux métiers de l'habitat écologique, de la construction soutenable ou du recyclage (valorisation des déchets, utilisation des ressources et matériaux locaux) qui sauront tirer profit des systèmes BIM et CIM en vue d'améliorer les écosystèmes territoriaux et les conditions de vies des habitants.

\subsection{Villes ouvertes et droit à la ville : coopération et contribution des habitants}

Il s'agirait ainsi de réactiver le projet d'une «ville ouverte ${ }^{34}$ » à l'époque des technologiques numériques: en parlant de «ville ouverte», R. Sennett montre que l'inachèvement ou de l'incomplétude des infrastructures constitue une condition de possibilité de l'implication des habitants et du dynamisme urbain. Sennett invite en effet à envisager la ville comme un système ouvert, susceptible de se transformer dans le temps et d'accueillir des événements improbables (contrairement aux systèmes fermés, caractérisés par leur surdétermination fonctionnelle, leur intégration, leur homogénéité et leur prédictibilité). Le design de la «ville ouverte » doit pour cela mettre en œuvre les formes architecturales incomplètes ou inachevées, modifiables au cours du temps en fonction des besoins des habitants et par ces mêmes habitants : les formes doivent pouvoir se transformer avec les fonctions des bâtiments, devenant ainsi des structures évolutives et vivantes.

C'est précisément ce que pourraient permettre les démarches BIM et CIM, qui permettent de suivre les évolutions des bâtiments et des infrastructures en temps réel, ainsi que d'évaluer les usages qui en sont faits par les habitants : à condition de s'inscrire dans des projets coordonnés par les institutions territoriales, ces données pourraient faire l'objet de délibération citoyenne et constituer ainsi le support de prise des décisions collectives, concernant l'aménagement et la gestion urbaine. Il s'agirait ainsi de transformer un rapport de force à long terme en capacitant des habitants sur ces questions et en demandant à des acteurs ayant des conceptions et des pratiques de la ville tout à fait différents d'en imaginer fertilement de nouveaux contours (notamment les jeunes générations, «post-digital natives » à la fois grands consommateurs d'applications et préoccupés par les enjeux économiques et écologiques à venir).

\footnotetext{
${ }^{32}$ Chauder B., Pastrascu M., Bouillon J.-L., « La maquette numérique dans le secteur du bâtiment. ", Revue française des sciences de l'information et de la communication, 9, 2016.

${ }^{33}$ B. Stiegler (2018). « New Urban Engineering, new Urban Genius », Lecture in Shangai.

${ }^{34}$ R. Sennett, « The open city » : http://richardsennett.com/site/senn/UploadedResources/The\%200pen\%20City.pdf
} 
Les technologies numériques et les logiciels BIM et CIM pourraient alors devenir les supports de pratiques de « coopération $^{35}$ » (Sennett) ou de « contribution $^{36}{ }$ (Stiegler), c'est-à-dire, d'activités collectives au sein desquelles les individus s'associent pour faire face aux difficultés du quotidien et pour imaginer des solutions, constituant ainsi des communautés de savoirs (savoir-faire, savoir vivre, savoirs techniques, savoirs théoriques) qui font la richesse des territoires. Ce sont donc de nouveaux savoirs de la ville qu'il s'agit de constituer, en favorisant les projets de recherche interdisciplinaires et d'expérimentations territoriales, et afin de constituer un nouveau «génie urbain ${ }^{37}$ » distribué sur le territoire. Il s'agirait ainsi de concrétiser un « droit à la ville numérique », en donnant aux habitants (et notamment aux jeunes générations) la capacité de comprendre les nouveaux milieux urbains et de devenir acteurs de la transformation industrielle en cours, à travers la pratique de nouveaux savoirfaire, savoir-vivre et savoirs théoriques locaux, susceptibles d'orienter les développements technologiques à venir - et d'éviter ainsi les services standardisés et la «souveraineté fonctionnelle » des smart cities.

\subsection{Education technologique et culture technique à l'époque du numérique}

Pour ce faire, la simple utilisation des outils BIM ou CIM par les professionnels ne peut suffire : pour ne pas devenir de simples moyens au service d'un pouvoir technocratique, mais des objets de connaissance, de réflexion et d'appropriation par les habitants, ceux-ci doivent être pratiquées dans le cadre de projets collectifs, et non pas seulement utilisées ou consommés par des individus incapables de les déchiffrer. Or, pour pouvoir s'approprier ces nouvelles technologies, participer à des démarches véritablement coopératives avec les acteurs professionnels et être porteurs de nouvelles initiatives, les habitants doivent eux-mêmes disposer d'une culture (à la fois savante, technique et pratique) de ces nouveaux instruments numériques et des transformations de l'urbanité qu'ils engendrent.

Comme le soulignait déjà le philosophie Gilbert Simondon dans les années $1960^{38}$, une telle culture technique est aujourd'hui absente de la plupart des programmes scolaires classiques : c'est la raison pour laquelle il semble nécessaire d'impliquer les institutions éducatives et académiques dans des projets de recherche contributive, associant les plus jeunes générations aux professionnels de l'urbanisme, de l'architecture et de la construction et aux chercheurs académiques spécialistes de ces questions.

De plus, tout porte à croire que cette adoption des technologies urbaines numériques pourrait s'effectuer via la pratique de certains jeux vidéos : en effet, une fois configuré selon un cadre technique approprié, un jeu vidéo de construction Minecraft pourrait permettre d'introduire des mécaniques de jeu identifiables à une démarche CIM (modélisation 3D d'un territoire à partir de cartes IGN, règles de construction selon des données territoriales, articulation des divers corps de métiers, coopération des acteurs/joueurs, etc.). Le jeu vidéo pourrait en effet s'articuler avec des logiciels de modélisation utilisés par les professionnels afin de permettre de capaciter les jeunes générations (futurs habitants) aux nouvelles techniques de modélisation, de construction et de gestion urbaine. Il devrait alors être pratiqué en parallèle à des enquêtes de terrain, permettant aux joueurs de s'approprier activement leur territoire et ses transformations technologiques et urbanistiques, à travers un agencement entre espace virtuel et réalité géographique, comme l'ont déjà expérimenté certaines associations ${ }^{39}$. Les travaux sur

\footnotetext{
${ }^{35}$ Voir R. Sennett, Ensemble. Pour une éthique de la coopération, Paris, Albin Michel, 2014 et R. Sennett, Bâtir et habiter. Pour une éthique de la ville, Paris, Albin Michel, 2019.

${ }^{36}$ B. Stiegler, La société automatique. t.1 L'avenir du travail., Paris, Fayard, 2015.

${ }^{37}$ C. Martinand, « Pour un génie urbain problématique », Les Annales de la recherche urbaine, N14, 1982.

${ }^{38}$ G. Simondon, «Place d'une initiation technique dans une formation humaine complète » (1953) in Sur la technique, Paris, PUF, 2014.

${ }^{39}$ Sur ce point, voir les travaux de l'association 3Hit Combo dans le cadre du projet RennesCraft : https://rennes2030.fr/le-projeturbain/on-en-parle/la-concertation-en-2016/les-ateliers-rennescraft/ 
le jeu s'articuleraient alors avec des travaux sur des fonds de dossiers d'aménagements (plans, cartes, enquêtes publiques, cahier des charges, historique, maquettes physiques et numériques, plans d'urbanisme et d'architecture, étude des coûts, données BIM) permettant aux enseignants et aux élèves, accompagnés par des équipes de professionnels et de chercheurs associés, de construire un projet urbain en partant des méthodes et outils professionnels, complétés par une démarche ouverte et contributive.

Ce que Simondon décrivait comme une «initiation technique ${ }^{40}$ » pourrait ainsi se constituer, permettant aux jeunes générations d'intérioriser un certain nombre de «formes techniques » ou de «schèmes de technicité fondamentaux », qui les rendent capables de comprendre, de manipuler et éventuellement de transformer les technologies du BIM. Mais Simondon veillait aussi à distinguer l'éducation technologique de simples « travaux manuels », en insistant sur sa dimension réflexive et culturelle : les travaux manuels ne sont pas bons en eux-mêmes et comme divertissements dépourvus d'intellectualité, ils n'ont de sens que dans le cadre «d'une technologie approfondie, liée à l'histoire de la pensée et à la conscience de la société », permettant aux jeunes générations d'hériter du " patrimoine technique de l'humanité » et de saisir la continuité des inventions techniques ${ }^{41}$, depuis les villes néolithiques jusqu'aux dites «smart cities». L'initiation technique aux technologies BIM (via la pratique du jeu vidéo éventuellement) devrait donc s'articuler au développement d'une conscience historique et technique de l'urbanité, permettant aux futurs citoyens d'appréhender les enjeux anthropologiques, économiques et politiques de la nouvelle révolution urbaine, et de prendre la mesure des dynamiques industrielles en cours. Le développement d'une telle culture urbaine critique implique une démarche transdisciplinaire qui pourrait engager les enseignants à problématiser leurs disciplines académiques autour de la question de l'urbanitéf ${ }^{42}$ dans le cadre de projets collectifs, afin de former les citoyens de la ville connectée et automatisée de demain.

A titre d'exemple, en philosophie, le rôle de la ville dans la formation de la pensée rationnelle pourrait être envisagé ; en histoire et géographie, les enseignements pourraient se focaliser sur l'histoire et la géographie urbaine, l'histoire du quartier et de la ville ; en littérature et français, des travaux pourraient être effectués autour de l'écriture de la ville, à travers l'étude de textes littéraires et techniques; en mathématiques, la question des ratios, des rapports, des statistiques ou de la fabrique des algorithmes pourraient être mobilisée ; en physique, l'étude des matériaux de construction semble fondamentale; en sciences de la vie et de la terre, l'étude des données environnementales et des écosystèmes locaux; en sciences économiques et sociales, l'étude des modèles économiques, des rapports entre public et privé ; etc.

\section{Conclusion}

Si les nouvelles technologies urbaines présentent le risque d'une automatisation de la gestion urbaine et d'une standardisation des formes architecturales et des modes de vies urbains, elles ouvrent aussi de nombreuses potentialités pour la constitution d'une nouvelle intelligence urbaine et collective

\footnotetext{
${ }^{40}$ G. Simondon, "Place d'une initiation technique dans une formation humaine complète » (1953) in Sur la technique, Paris, PUF, 2014.

${ }^{41}$ G. Simondon, «Place d'une initiation technique dans une formation humaine complète » (1953) in Sur la technique, Paris, PUF, 2014.

${ }^{42}$ A titre d'exemple, on pourrait envisager le rôle de la ville dans la formation de la pensée rationnelle en philosophie, en histoire et géographie, on pourrait se focaliser sur l'histoire et la géographie urbaine, l'histoire du quartier et de la ville; en littérature et français, des travaux pourraient être effectués autour de l'écriture de la ville, à travers l'étude de textes littéraires et techniques ; en mathématiques, la question des ratios, des rapports, des statistiques ou de la fabrique des algorithmes pourraient être mobilisée ; en physique, l'étude des matériaux de construction semble fondamentale; en SVT, l'étude des données environnementales et des écosystèmes locaux; en sciences économiques et sociales, l'étude des modèles économiques, des rapports entre public et privé ; etc.
} 
du numérique, fondée sur la contribution active des habitants et sur des milieux urbains susceptibles de développer leurs capacités. Il nous semble que la démarche de City Information Modeling/Management et les technologies de Building Information Modeling/Management, en tant qu'elles permettent une représentation numérique partagée d'un objet bâti ou d'un espace urbain afin de faciliter la mise en relation des différents acteurs dans les processus de conception, de construction, de maintenance ou d'exploitation, sont porteuses de potentialités contributives, qui demandent à être développées, afin de permettre la constitution d'habitats et de milieux urbains contributifs, c'est-à-dire fondés sur la participation active des habitants à la production de l'espace urbain, et sur le développement de leurs savoirs (savoir-faire techniques, savoir-vivre citadins et savoirs théoriques scientifiques, juridiques, politiques, architecturaux, urbanistiques etc.).

Les professionnels (urbanistes, architectes, ingénieurs, maîtres d'ouvrage, consultants informatique, entreprises) ont tout à gagner de la démocratisation de ces outils : celle-ci leurs permettra d'inventer les nouveaux métiers et les nouveaux savoirs qui sont à l'horizon de ces transformations technologiques, et d'inventer de nouvelles manières de travailler ensemble et de nouveaux modèles économiques pour lutter contre les risques de l'automatisation et de la disruption. Les communautés de savoir constituées d'habitants, de professionnels et d'acteurs économiques et politiques pourraient constituer la base d'une nouvelle puissance publique territoriale. Il s'agirait alors de substituer au discours promotionnel des smart cities le développement de villes véritablement intelligentes, c'est-à-dire, fondée sur la coopération ${ }^{43}$ et les capacités ${ }^{44}$ des habitants, et renforçant la soutenabilité écologique et la solvabilité économique des territoires, ainsi que la délibération collective des citoyens. Les habitants qui avaient été réduits au statut d'usagers et de consommateurs de services deviendraient ainsi chercheurs et prescripteurs, capables de délibérer, de débattre et de discuter avec les acteurs industriels et les responsables politiques, pour proposer des dispositifs technologiques et des organisations sociales favorisant un développement urbain soutenable, habitable et désirable.

\section{Bibliographie}

[AKR 87] AKRICH M., « Comment décrire les objets techniques ?», Techniques et culture, 49-64, 1987.

[ARA 15] ARAYA D., Smart Cities as Democratic Ecologies, Palgrave, London-New York, 2015.

[BAU 19] Bauwens M. et al., Peer to Peer: The Commons Manifesto, University of Westminster Press, London, 2019.

[BER 02] BERQUE A., «L'habitat insoutenable Recherche sur l'histoire de la désurbanité », in L'Espace géographique, (tome 31), p. 241-251, 2002.

[BRAU 77] BRAUDEL F., La dynamique du capitalisme, Flammarion, Paris, 1977.

[CAR 14] CARTA M., Reimagining urbanism. Creative, smart and Green Cities for the changing times, ListLab: Barcelona-Trento, 2014.

[CHAU 16] Chauder B., PASTRASCU M., Bouillon J.-L., « La maquette numérique dans le secteur du bâtiment. », Revue française des sciences de l'information et de la communication, 9, 2016.

[COU 18] Courmont A., « Plateforme, big data et recomposition du gouvernement urbain. Les effets de Waze sur les politiques de régulation du trafic. », Revue française de sociologie, 59(3), 423, 2018.

[CRI 18] CRISTIA E., ZALIO P.-P. et GUENA F., «Quand le BIM met la maquette à l'épreuve du numérique » in SCAN’18, Nantes, SHS Web of Conferences, Nantes, 2018.

[CRI 18] CRISTIA E., ZALIO P.-P. et GUENA F., «Fabriquer une maquette numérique BIM et reconsidérer le projet architectural », in Le BIM entre recherche et industrialisation, Eyrolles, Paris, 2019.

[DUP 07] DUPUY G., L’informatisation des villes, PUF, Paris, 2007.

\footnotetext{
${ }^{43}$ Sur la nécessité sociale de la coopération, et sur les liens entre coopération et urbanité, voir R. Sennett, Ensemble. Pour une éthique de la coopération, Paris, Albin Michel, 2014 et Bâtir et habiter. Pour une éthique de la ville, Paris, Albin Michel, 2019.

${ }^{44}$ Sur la valeur économique des «capabilités », voir A. Sen, Repenser l'inégalité, Paris, Points, 2012 et Ethique et économie, Paris, PUF, 2012.
} 
[HER 07] HERRENSCHMIDT C., Les Trois Écritures, Langue, nombre, code, Gallimard, Paris, 2007.

[HAL 17] HalPern O., Mitchell R. et GeOGHEGan B., «The smartness mandate. Notes towards a critique », Grey Room, $\mathrm{N}^{\circ}$ 68, p.106-12, 2017.

[MAN 93] Mangematin V., "Compétition technologique : les coulisses de la mise sur le marché. », Annales des Mines, 1993.

[MAR 82] MARTINAND C., «Pour un génie urbain problématique », Les Annales de la recherche urbaine,

$\mathrm{N}^{\circ} 14,1982$.

[MOR 15] MOROZOV E., « Our cities shouldn’t rely on Uber to devise new transport choices », The Guardian, 2015.

[MOR 15] MOROZOV E. et BRIA F., Ripensare la smart city, Codice Ed, Torino, 2015.

[MOR 13] MOROZOv E., Pour tout résoudre, cliquez ici. Les aberrations du solutionnisme technologique., FYP Editions, Paris, 2013.

[LEF 68] LeFEBVRE H., Le droit à la ville. Espace et politique., Seuil, Paris, 1968.

[PAS 15] PASQUALE F., The black box society: the secret algorithms that control money and information, Harvard University Press, London, 2015.

[PAS 17] PASQUALE F. «From Territorial to Functional Sovereignty: The Case of Amazon. », Public Policy, 2017.

[PIC 16] PICON A., « L’avènement de la ville intelligente. », Sociétes, n¹32(2), 9-24, 2016.

[ROU 13] ROUVROY A. et BERNS T., "Gouvernementalité algorithmique et perspectives d'émancipation. Le disparate comme condition d'individuation par la relation ?», Réseaux, vol. 177, no. 1, pp. 163-196, 2013.

[SAD 19] SADOWSKI J., « The Captured City. The "smart city" makes infrastructure and surveillance indistinguishable », Real Life, 12 November 2019, 18 November 2019.

[SEN 92] SEN A., Inequality Re-Examined, Harvard University Press, Cambridge, Massachusetts, 1992.

[SEN 14] SENNETT R., Ensemble. Pour une éthique de la coopération, Albin Michel, Paris, 2014.

[SEN 19] SENNETT R., Bâtir et habiter. Pour une éthique de la ville, Albin Michel, Paris, 2019.

[SIM 58] SimOndon G., Du mode d'existence des objets techniques, Jérôme Million, Paris, 1958.

[SIM 14] SimOndon G., Sur la technique, PUF, Paris, 2014.

[STI 15] STIEGLER B., La société automatique. t. 1 L'avenir du travail, Fayard, Paris, 2015.

[STI 18] STIEGLER B., « New Urban Engineering, new Urban Genius », unpublished lecture in Shangai, 2018.

[SUP 15] SUPIOT A., La gouvernance par les nombres, Fayard, Paris, 2015.

[VEL 19] Velgue P., «Lire la Chine », Perspectives chinoises [En ligne], Centre d'étude français sur la Chine contemporaine, 2019. 